\title{
ON THE INVERSE PROBLEM OF GALOIS THEORY OF DIFFERENTIAL FIELDS
}

\section{A. BIALYNICKI-BIRULA}

0. One can ask what algebraic groups are isomorphic to groups of automorphism of strongly normal extensions of a fixed ordinary differential field (see [2]). The purpose of the note is to give a contribution in this direction. We shall prove the following theorem.

TheOREM. Let $\mathcal{F}$ be an ordinary differential field with algebraically closed field of constants $C$ and suppose that $\mathcal{F}$ is of finite transcendence degree over $C$ but is different from $C$. Let $G$ be a connected nilpotent affine algebraic group defined over $C$. Then there exists a strongly normal extension $\mathcal{E}$ of $\mathcal{F}$ such that the Galois group $\mathcal{G}(\mathcal{E} / \mathcal{F})$ is isomorphic to $G(C)$.

1. All fields considered here are of characteristic 0 . Let $F$ be a field, let $C$ be an algebraically closed subfield of $F$. Let $G$ be a connected algebraic group defined over $C . F(G)$ denotes the field of all rational functions on $G$ defined over $F$. If $g \in G$ then $F(g)$ denotes the field generated by $g$ over $F$. We shall say that a derivation of $F(G)$ commutes with $G^{*}(C)$ if it commutes with $g^{*}$, for every $g \in G(C)$, where $g^{*}$ denotes the automorphism of $F(G)$ induced by the left translation by $g$, i.e., $\left(g^{*} f\right)(x)=f(g x)$, for any $x \in G$. $\left(S_{F}\right.$ denotes the Lie algebra of all derivations of $F(G)$ that are zero on $F$ and which commute with $G^{*}(F)$. If $G_{1}$ is a normal subgroup of $G$ defined over $F$ then $F\left(G / G_{1}\right)$ is canonically isomorphic to a subfield of $F(G)$; we shall identify $F\left(G / G_{1}\right)$ and this subfield.

If $R$ is an integral domain then $(R)$ denotes the field of fractions of $R$. Every derivation $d$ of $R$ can be uniquely extended to a derivation of $R$ (the extended derivation will be also denoted by $d$ ). If $F_{1}, F_{2}$ are two fields containing $F$ as a subfield and if $d_{1}, d_{2}$ are derivations of $F_{1}, F_{2}$, respectively, such that $d_{1}\left|F=d_{2}\right| F$ and $d_{1}(F) \subset F$ then $d_{1} \otimes d_{2}$ denotes the derivation of $F_{1} \otimes_{F} F_{2}$ determined by $\left(d_{1} \otimes d_{2}\right)(a \otimes b)$ $=d_{1}(a) \otimes b+a \otimes d_{2}(b)$, for every $a \in F_{1}$ and $b \in F_{2}$.

$d_{0}$ denotes the zero derivation of a field (it will be always clear what field we have in mind). The underlying field of an ordinary differential field $\mathcal{F}$ will be denoted by $F$.

2. Lemma 1. If $d_{1}$ belongs to the center of $\mathbb{S}_{c}$ then the derivation $d_{1} \otimes d_{0}$ of $(C(G) \otimes F) \quad(=F(G))$ commutes with every derivation $d$ of $F(G)$ such that $d(F) \subset F$ and $d g^{*}=g^{*} d$ for every $g \in G(C)$.

Received by the editors June 12, 1963. 
Proof. Let $d$ be as in the lemma. Then $d-d_{0} \otimes(d \mid F)$ is zero on $F$ and commutes with $G^{*}(F)$ and so $d-d_{0} \otimes(d \mid F) \in\left(\mathcal{S}_{F}\right.$. But $d_{1} \otimes d_{0}$ belongs to the center of $\mathcal{S}_{F}$ and commutes with $d_{0} \otimes(d \mid F)$. Thus $d_{1} \otimes d_{0}$ commutes with $d=d-d_{0} \otimes(d \mid F)+d_{0} \otimes(d \mid F)$.

Lemma 2. Let $G_{1}$ be a normal subgroup of $G$ defined over $C$ and let $d^{0}$ be a derivation of $F\left(G / G_{1}\right)$ such that $d^{0}(C)=0$ and $d^{0}$ commutes with any element from $\left(G / G_{1}\right)^{*}(C)$. Then there exists an extension $d^{\prime}$ of $d^{0}$ to a derivation of $F(G)$ that commutes with $G^{*}(C)$.

Proof. Let $g$ be a generic point of $G$ over $F(G)$. Extend $d^{0}$ to a derivation $d_{1}$ of $F(G)$ and let $d_{2}$ be the extension of $d_{1}$ to a derivation of $F(g)(G)$ which is trivial on $C(g)$. Let $V$ be a nonempty affine open subset of $G$ defined over $C$ and let $C\left[x_{1}, \cdots, x_{n}\right]$ be the coordinate ring of $V$ over $C$. Then there exists $h_{0} \in V(C)$ such that $d_{1} x_{1}, \cdots, d_{1} x_{n}$ are defined at $h_{0}$. Hence, if $a \in F(g)(G)$ is defined at $h_{0}$ then $d_{2}(a)$ is also defined at $h_{0}$. In particular, for any $a \in F(G), d_{2}\left(\left(g h_{0}^{-1}\right)^{*} a\right)$ is defined at $h_{0}$ (since $\left.\left(\left(g h_{0}^{-1}\right)^{*} a\right)\left(h_{0}\right)=a(g)\right)$. Let, for any $a \in F(G), d^{\prime}(a)$ be the element of $F(G)$ such that $d^{\prime}(a)(g)=d_{2}\left(\left(g h_{0}^{-1}\right)^{*} a\right)\left(h_{0}\right)$. One can easily see that the definition of $d^{\prime}$ does not depend on $g$. In particular, if $g_{1}$ is any point of $G$ such that $C\left(g_{1}\right)=C(g)$, then $g_{1}$ is generic for $G$ over $F$ and so $d^{\prime}(a)\left(g_{1}\right)=d_{2}\left(\left(g_{1} h_{0}^{-1}\right)^{*} a\right)\left(h_{0}\right)$. Hence, for any $h \in G(C)$ $\left(h^{*} d^{\prime}(a)\right)(g)=d^{\prime}(a)(h g)=d_{2}\left(\left(h g h_{0}^{-1}\right) * a\right)\left(h_{0}\right)=d_{2}\left(\left(g h_{0}^{-1}\right)^{*} h^{*} a\right)\left(h_{0}\right)$ $=d^{\prime}\left(h^{*} a\right)(g)$, since $C(h g)=C(g)$. Thus $d_{1} h^{*}=h^{*} d_{1}$, i.e., $d_{1}$ commutes with $G(C)^{*}$. Moreover, $d^{\prime}$ is a derivation of $F(G)$. Indeed

$$
\begin{aligned}
d^{\prime}(a+ & b)(g) \\
& =d_{2}\left(\left(g h_{0}^{-1}\right)^{*}(a+b)\right)\left(h_{0}\right)=d_{2}\left(\left(g h_{0}^{-1}\right)^{*} a\right)\left(h_{0}\right)+d_{2}\left(\left(g h_{0}^{-1}\right) b\right)\left(h_{0}\right) \\
& =d^{\prime}(a)(g)+d^{\prime}(b)(g)
\end{aligned}
$$

and

$$
\begin{aligned}
d^{\prime}(a b)(g) & =d_{2}\left(\left(g h_{0}^{-1}\right)^{*} a b\right)\left(h_{0}\right) \\
= & d_{2}\left(\left(g h_{0}^{-1}\right)^{*} a\right)\left(h_{0}\right) \cdot\left(g h_{0}^{-1}\right)^{*} b\left(h_{0}\right)+\left(g h_{0}^{-1}\right)^{*} a\left(h_{0}\right) \cdot d_{2}\left(\left(g h_{0}^{-1}\right)^{*} b\right)\left(h_{0}\right) \\
= & d^{\prime}(a)(g) \cdot b(g)+a(g) \cdot d^{\prime}(b)(g) .
\end{aligned}
$$

Finally, if $a \in F\left(G / G_{1}\right)$ then

$$
\begin{aligned}
d^{\prime}(a)(g) & =d_{2}\left(\left(g h_{0}^{-1}\right)^{*} a\right)\left(h_{0}\right)=d^{0}\left(\left(g h_{0}^{-1}\right)^{*} a\right)\left(h_{0}\right) \\
& =\left(g h_{0}^{-1}\right)^{*} d^{0}(a)\left(h_{0}\right)=d^{0}(a)(g),
\end{aligned}
$$

i.e., $d^{\prime}$ is an extension of $d^{0}$. This completes the proof of the lemma.

Lemma 3. Let $G_{1}$ be a connected central one-dimensional normal sub- 
group of an affine connected algebraic group $G$, both defined over $F$. Let $d_{1} \in \oiint_{F}$ be a derivation in the direction of $G_{1}$. Then, for any $a \in F(G)$, $d_{1}(a)=0$ if and only if $a \in F\left(G / G_{1}\right)$. Moreover, there exists an element $b \in F(G)-F\left(G / G_{1}\right)$ such that either $d_{1}(b)=c \cdot b$ or $d_{1}(b)=c$, where $c$ is an element from $F\left(G / G_{1}\right)$.

Proof. The first part of the lemma is well known. Let $b^{\prime}$ be a regular function on $G$ such that $b^{\prime} \in F(G)-F\left(G / G_{1}\right)$. Then $G_{1}^{*}(F) \cdot b^{\prime}$ generates a finite-dimensional $F$-vector space. Since $G_{1}$ is one-dimensional and connected, hence we may assume that this space is either one-dimensional or two-dimensional with basis $b_{0}, b^{\prime}$, where $b_{0}$ $\in F\left(G / G_{1}\right)$ and $g^{*}\left(b^{\prime}\right)=\alpha(g) b_{0}+b^{\prime}, \quad \alpha \in F\left(G / G_{1}\right)$ and $\alpha(g) \neq 0$ if $g \neq$ identity $e$ of $G_{1}$. Then it follows from Lemma $7[1]$ that in the first case $d_{1}\left(b^{\prime}\right)=c b^{\prime}$, where $c$ is an element from $F\left(G / G_{1}\right)$ and we may take $b=b^{\prime}$. In the second case (again by Lemma $\left.7[1]\right) c_{1} d_{1}^{2}\left(b^{\prime}\right)+c_{2} d_{1}\left(b^{\prime}\right)$ $+c_{3} b^{\prime}=0$, for some $c_{1}, c_{2}, c_{3} \in F\left(G / G_{1}\right)$ which do not vanish simultaneously. Then $0=g^{*}\left(c_{1} d_{1}^{2}\left(b^{\prime}\right)+c_{2} d_{1}\left(b^{\prime}\right)+c_{3} b^{\prime}\right)=c_{1} d_{1}^{2}\left(b^{\prime}\right)+c_{2} d_{1}\left(b^{\prime}\right)$ $+c_{3}\left(\alpha(g) b_{0}+b^{\prime}\right)$, for every $g \in G_{1}(F)$. Hence $c_{3}=0$ and $c_{1} \neq 0$. If $c_{2} \neq 0$, then $d_{1}\left(d_{1}\left(b^{\prime}\right)\right)=-c_{2} / c_{1}, d_{1}\left(b^{\prime}\right) \neq 0$, and we take $b=d_{1}\left(b^{\prime}\right)$. If $c_{2}=0$, then $d_{1}^{2}\left(b^{\prime}\right)=0$. Hence $d_{1}\left(b^{\prime}\right) \in F\left(G / G_{1}\right)$, and we take $b=b^{\prime}$.

Lемма 4. Let $\mathcal{F}$ be an ordinary differential field with derivation d, let $C$ be the field of constants of $\mathcal{F}$ and suppose that $\mathcal{F}$ is of finite transcendence degree over $C$. Let $\mathcal{F}_{1}$ be a (differential) subfield of $\mathcal{F}$ which is not contained in $C$ and let $c \in C$. Then there exist $a_{1}, a_{2} \in F_{1}, a_{1} \neq 0 \neq a_{2}$, such that there is no element $y \in \mathcal{F}-C$ which satisfies either $d y=a_{1} \cdot c$ or $d y=a_{2} c y$.

Proof. We may suppose that $F_{1}$ contains an element $x$ such that $d x=1$ (let $x \in F_{1}-C$; then $d x \neq 0$ and we may replace $d$ by $1 / d x \cdot d$ ). If $d y_{n}=c /(x+n), y_{n} \in \mathcal{F}-C$, where $n$ is an integer, then, one can prove that the elements $y_{i}$, for different integers $i$, are algebraically independent over $C$. Similarly, if $d z_{n}=x^{n} c z_{n}$, then the elements $z_{i}$, for different integers $i$, are also algebraically independent over $C$. Hence $F$ contains only a finite number of elements $y_{i}$ and $z_{i}$. Thus, for some $n, y_{n}, z_{n} \notin \mathcal{F}$ and the lemma is proved.

3. Proof of the theorem. Let $d$ be the nonzero derivation of $F$. We shall show that one can extend $d$ to a derivation $d^{*}$ of $F(G)$ which commutes with $G^{*}(C)$ and has $C$ as the field of constants. Proof by induction on the dimension of $G$.

If $\operatorname{dim} G=0$, then this is trivial.

Suppose that the above is true for connected nilpotent affine groups of dimension $n$ and let $\operatorname{dim} G=n+1$. There exists a central connected 
normal subgroup $G_{1}$ of $G$ defined over $C$ and of dimension 1. Then $G / G_{1}$ is an affine nilpotent connected group of dimension $n$. Hence there exists an extension $d^{0}$ of $d$ to a derivation of $F\left(G / G_{1}\right)$ such that $C$ is the field of constants of $d^{0}$ and $d^{0}$ commutes with $\left(G / G_{1}\right)^{*}(C)$. It follows from Lemma 2 that $d^{0}$ can be extended to a derivation $d^{\prime}$ of $F(G)$ that commutes with $G^{*}(C)$. Let $d_{1} \in B_{F}$ be a derivation of $F(G)$ in the direction of $G_{1}$. Then the field of constants of $d_{1}$ is $F\left(G / G_{1}\right)$ and it follows from Lemma 1 that $d_{1}$ commutes with every derivation $a d^{\prime}$, where $a \in F$. Therefore the set of all $b \in F(G)$, for which $d_{1}(b)$ $=a d^{\prime}(b)$, where $a$ is fixed, is a subfield $F_{a}$ of $F(G)$ closed under $d_{1}$ (and $a d^{\prime}$ ). Indeed, it is easy to see that this is a field. Moreover, if $d_{1}(b)=a d^{\prime}(b)$, then $d_{1}\left(d_{1}(b)\right)=d_{1}\left(a d^{\prime}(b)\right)=a d^{\prime}\left(d_{1}(b)\right) . C$ is the field of constants of $d_{1} \mid F_{a}$ for $a \neq 0$, since the field of constants of $d_{1}$ is $F\left(G / G_{1}\right)$ and the field of constants of $a d^{\prime} \mid F\left(G / G_{1}\right)$ is $C$. And we want to prove that $F_{a}=C$, for some $a \in F$. Let $a \in F$; consider the ordinary differential field $\left(F(G) \otimes_{c} F\left(G / G_{1}\right)\right)$ together with the derivation $a d^{\prime} \otimes d_{0}$ and the algebraic closure $\left(F(G) \otimes_{c} F\left(G / G_{1}\right)\right)^{*}$ of $\left(F(G) \otimes_{c} F\left(G / G_{1}\right)\right)$ with the unique extension $\left(a d^{\prime} \otimes d_{0}\right)^{*}$ of $\left(a d^{\prime} \otimes d_{0}\right)$. $F_{a}$ is linearly disjoint from $F\left(G / G_{1}\right)$ over $C$ since $F\left(G / G_{1}\right)$ is the field of constants of $d_{1}$ and $C$ is the field of constants of $d_{1} \mid F_{a}$ (see Proposition 1 in [3] or Lemma 1 in [1]). Hence there exists a subfield of $F(G)$ with $d_{1}$ which is canonically isomorphic to $\left(F_{a} \otimes_{c} F\left(G / G_{1}\right)\right)$ with $\left(a d^{\prime} \mid F_{a}\right) \otimes d_{0} . F(G)$ is an algebraic extension of the subfield unless $F_{a}=C$ and this isomorphism maps $b$ onto $1 \otimes b$, for every $b \in F\left(G / G_{1}\right)$. Therefore $F_{a} \neq C$ implies that there exists an isomorphism $\alpha_{a}$ of $F(G)$ with $d_{1}$ into $\left.(F(G)) \otimes_{c} F\left(G / G_{1}\right)\right)^{*}$ with $\left(a d^{\prime} \otimes d_{0}\right)^{*}$ such that $\alpha_{a}(b)=1 \otimes b$, for every $b \in F\left(G / G_{1}\right)$. It follows from Lemma 3 that there exist elements $c \in F\left(G / G_{1}\right)$ and $y \in F(G)-F\left(G / G_{1}\right)$ such that either $d_{1} y=c$ or $d_{1} y=c y$. Therefore, for every $a \in G, a \neq 0$ for which $F_{a} \neq C$, we have that either $\left(a d^{\prime} \otimes d_{0}\right)^{*} \alpha_{a}(y)=1 \otimes c$ or $\left(a d^{\prime} \otimes d_{0}\right)^{*} \alpha_{a}(y)$ $=(1 \otimes c) \alpha_{a}(y)$, i.e., either $\left(d^{\prime} \otimes d_{0}\right)^{*} \alpha_{a}(y)=1 \otimes c / a \otimes 1$ or $\left(d^{\prime} \otimes d_{0}\right)^{*} \alpha_{a}(y)$ $=1 \otimes c / a \otimes 1 \alpha_{a}(y)$. But it follows from Lemma 4 that there exist $a_{1}, a_{2} \in F$ such that neither $\left(d^{\prime} \otimes d_{0}\right) *_{z}=1 \otimes c / a_{1} \otimes 1$ nor $\left(d^{\prime} \otimes d_{0}\right) *_{z}$ $=\left(1 \otimes c / a_{2} \otimes 1\right) z$ has a solution $z$ in $\left(F(G) \otimes F\left(G / G_{1}\right)\right)^{*}$. Then $a_{1} \neq 0 \neq a_{2}$ and either $F_{a_{1}}=C$ or $F_{a_{2}}=C$. If $F_{a}=C$, then $a \neq 0$ and the field of constants of $d^{*}=(1 / a) d_{1}-d^{\prime}$ is $C$. Moreover, $d^{*}$ commutes with $G^{*}(C)$. Thus we have proved by induction that there exists an extension $d^{*}$ of $d$ that commutes with $G^{*}(C)$ and has $C$ as the field of constants.

Now if $d^{*}$ is such a derivation then $F(G)$ with $d^{*}$ is a strongly normal extension of $F$ and $G(C)$ is the Galois group of the extension (see Proposition 1 and Theorem 1 in [1]). 


\section{REFERENCES}

1. A. Bialynicki-Birula, On Galois theory of fields with operators, Amer. J. Math. 84 (1962), 89-109.

2. E. R. Kolchin, Galois theory of differential fields, Amer. J. Math. 75 (1953), 753-824.

3. - Algebraic matric groups and the Picard-Vessiot theory of homogeneous linear ordinary differential equations, Ann. of Math. (2) 49 (1948), 1-42.

University of California, Berkeley and

Institute of Mathematics, P.A.N., Poland

\section{ON A REALIZATION OF A COMPLEMENTED ALGEBRA}

\section{PARFENY P. SAWOROTNOW}

In this note we intend to show that each simple complemented algebra is isomorphic to an algebra described in the example below (as in [6] we use the term "simple" to mean "simple and semisimple"). This paper can be considered as a continuation of [5] and [6].

In the example below (and in the proof of the theorem after it) we use terms "summable" and "integrable" in the sense defined in Chapter III of [3].

ExAmple. Let $(S, \mu)$ be a measure space. Let $K(s)$ be a real-valued function defined on $S$ and having the following properties:

(i) $K(s)$ is finite almost everywhere,

(ii) there exists a positive number $a$ such that $a \leqq K(s)$ for each $s \in S$,

(iii) the restriction of $K(s)$ to any summable subset of $S$ is integrable (in particular $K(s)$ may be integrable).

Let $A$ be the set of all complex-valued members $x$ of $L^{2}(S \times S, \mu \times \mu)$ such that $\iint|x(t, s)|^{2} K(s) d t d s$ is finite. Then $A$ is a complemented algebra in the scalar product $(x, y)=\iint x(t, s) \bar{y}(t, s) K(s) d t d s$ and the multiplication $(x y)(t, s)=\int x(t, r) y(r, s) d r$ (we consider pointwise addition and pointwise multiplication with a scalar). If $K(s)$ is bounded above then $A$ is well complemented. Condition (ii) implies continuity of the multiplication (in both factors simultaneously); if $a=1$ then $\|x y\| \leqq\|x\|\|y\|$.

Presented to the Society, August 24, 1956, under the title Diagonalization of a complemented algebra; received by the editors July 15, 1963. 